\title{
Alteration of the immune status of umbilical cord mesenchymal stem cells stimulated by TLR1/2 agonist, Pam3Csk
}

\author{
PINGXI WANG ${ }^{1}$, FANWEI ZENG $^{1}$, LINA HE $^{2}$, JIN WANG $^{1}$, TINGJIU ZHANG ${ }^{1}$ and DONG ZHANG ${ }^{1}$ \\ Departments of ${ }^{1}$ Orthopedic Surgery and ${ }^{2}$ Pharmacy, The Central Hospital of Dazhou, Dazhou, Sichuan 635000, P.R. China
}

Received July 17, 2015; Accepted May 27, 2016

DOI: $10.3892 / \mathrm{mmr} .2016 .5520$

\begin{abstract}
Mesenchymal stem cells (MSCs) have been widely used in clinical trials due to their multiple differentiation ability, low immunogenicity and immunosuppressant effects on immune response. However, accumulating evidence has indicated that MSCs may stimulate in vivo immune responses and result in the disappearance of MSCs following engrafting. Toll-like receptors (TLRs) are important in immune response induction against invaded pathogens, however, the function of TLRs in regulating the immune status of MSCs has been seldom reported. The present stimulated umbilical cord (UC) MSCs by treatment with the TLR1/2 agonist, Pam3Csk, the to determine whether activation of TLR $1 / 2$ signaling alters the immune status of UCMSCs. The results indicated that activation of TLR1/2 increased the proliferation of peripheral blood mononuclear cells (PBMCs) and the production of lactate dehydrogenase in a PBMC-MSC co-culture system. The study also demonstrated that Pam3Csk induced the secretion of pro-inflammatory molecules, and increased the expression levels of cytokine and chemokines in UCMSCs. Flow cytometry analysis indicated that the levels of surface co-stimulators, CD80 and CD86, were increased on UCMSCs in the presence of Pam3Csk, whereas activation of TLR1/2 exerted no observable effect on the differentiation abilities of UCMSCs. The results of the current study indicated that activation of TLR $1 / 2$ signaling may alter the immune status of UCMSCs, however, further mechanistic research is required in future studies.
\end{abstract}

\section{Introduction}

Mesenchymal stem cell (MSC)-based therapy has been widely used in clinical trials and led to exciting developments in cell therapy, including the prevention of graft versus

Correspondence to: Professor Dong Zhang, Department of Orthopedic Surgery, The Central Hospital of Dazhou, 56 Nanyue miao Street, Dazhou, Sichuan 635000, P.R. China E-mail:dzsgkw@126.com

Key words: umbilical cord mesenchymal stem cells, Toll-like receptor $1 / 2$, Pam3Csk, pro-inflammatory molecules, immune status host disease (1), reducing liver fibrosis (2), remodeling of broken bone (3) and treatment of cardiovascular diseases (4). An important characteristic of MSCs is the ability to differentiate into various cell types (5). Another feature of MSCs is the lack of co-stimulatory molecules including CD80, CD86 and HLA-II, which result in MSCs failing to induce immune responses (6). Additionally, MSCs also suppress the immune reactions mediated by $\mathrm{T}$ cells, B cells, natural killer (NK) cells, dendritic cells and complements (7-9). However, recent studies have indicated that although MSCs exhibit low-immunogenicity and immunosuppressant abilities, MSCs used as a therapeutic may be rejected or injured by the host immune system and eventually disappear in vivo (10-12).

Toll-like receptors (TLRs) are a family of pathogen-associated molecular patterns (PAMPs) involved in mediating immune responses induced by invading pathogens (13). There are 10 members in the human TLRs family, which recognize distinct microbial products from bacteria, viruses and fungi (14). The TLRs also important for in MSC functions, including increasing osteogenic differentiation (TLR3) (15), promoting MSCs migration (TLR5) (16) and inhibiting MSCs mediating immunosuppression (TLR4) (17). However, the importance of TLRs in regulating the immune status of MSC has not been widely investigated. A previous report demonstrated that TLR7 stimulates the immunogenicity of MSCs (18), whereas TLR3 and 4 did not alter the immune status of MSCs. However, TLR3 and TLR4 agonists enhanced the expression of various immune-associated molecules (19). To the best of our knowledge, no previous report has investigated the importance of TLR1/2 on the immune status of MSCs. The current study used MSCs isolated from umbilical cord (UC) and activated the TLR1/2 pathway using a specific agonist, aiming to determine whether the activation of TLR1/2 changes the immune status of MSCs.

\section{Materials and methods}

Culture and stimulation of MSCs. The MSCs from UC were provided by Sichuan Umbilical Cord Blood Stem Cell Bank (Chengdu, China). The UCMSCs were maintained at $37^{\circ} \mathrm{C}$ with $5 \% \mathrm{CO}_{2}$ in Dulbecco's modified Eagle's medium (Invitrogen; Thermo Fisher Scientific, Inc., Waltham, MA, USA) supplemented with $10 \%$ fetal bovine serum (Invitrogen; Thermo Fisher Scientific, Inc., Waltham, MA, USA) at $1 \times 10^{5}$ cells/well in a 6-well plate. 
Table I. Primers used for reverse transcription-polymerase chain reaction.

\begin{tabular}{lllc}
\hline Gene & \multicolumn{1}{c}{ Forward primer } & \multicolumn{1}{c}{ Reverse primer } & Gen bank no. \\
\hline IFN- $\beta$ & CAGCAATTTTCAGTGTCAGAAGCT & TCATCCTGTCCTTGAGGCAGT & M28622 \\
IL-6 & GACCCAACCACAAATGCCA & GTCATGTCCTGCAGCCACTG & M14584 \\
IL-8 & CTGGCCGTGGCTCTCTTG & CCTTGGCAAAACTGCACCTT & NM_000584 \\
IL-10 & GGTGATGCCCCAAGCTGA & TCCCCCAGGGAGTTCACA & U16720 \\
TNF- $\alpha$ & GGTGCTTGTTCCTCAGCCTC & CAGGCAGAAGAGCGTGGTG & M10988 \\
CCL5 & GACACCACACCCTGCTGCT & TACTCCTTGATGTGGGCACG & NM_002985 \\
MCP-1 & AGCAGAGGCTGGAGAGCTACA & GGGTCAGCACAGATCTCCTTGT & NM_006273 \\
MCP-3 & CCTCTCCTGCCTCATGCTTATT & CTCTGTCTCTGCATCATTTGTGAA & U58914 \\
IP10 & TGAAATTATTCCTGCAAGCCAA & CAGACATCTCTTCTCACCCTTCTTT & NM_001565 \\
MIP-1 & GACACCACACCCTGCTGCT & TACTCCTTGATGTGGGCACG & NM_002985 \\
Nanog & CCAAAGGCAAACAACCCACTT & CGGGACCTTGTCTTCCTTTTT & NM_00129769 \\
Sox2 & CCCCTTTATTTTCCGTAGTTGTATTT & GATTCTCGGCAGACTGATTCAA & NM_003106.3 \\
Lin28 & GTCATCAGCGTCAGCAAAGG & CCCTGCTGCTCAGCACTT & NM_004235.4 \\
Otx2 & GGTTTCCTCTCCCTCTCCAC & AATTTGAATTTTACGTCTGCTG & NM_002448.3 \\
GAPDH & GAAGGTGAAGGTCGGAGTC & GAAGATGGTGATGGGATTTC & J04038 \\
\hline
\end{tabular}

IFN- $\beta$, interferon- $\beta$; IL, interleukin; TNF- $\alpha$, tumor necrosis factor- $\alpha$; CCL5, C-C motif chemokine ligand 5; MCP, monocyte chemoattractant protein; IP10, interferon $\gamma$-induced protein 10; MIP-1, macrophage inflammatory protein-1; Nanog, Nanog homeobox; SOX2, sex determining region Y-box 2; Lin28, Lin-28 homolog A; Otx2, orthodenticle homeobox 2.

TLR1/2 agonist, Pam3Csk, was purchased from Novus Biologicals, Ltd. (Cambridge, UK) and dissolved in sterile water to $0.5 \mathrm{mg} / \mathrm{ml}$ as the stock concentration. The final concentration of Pam3Csk used to stimulate UCMSCs was $100 \mathrm{ng} / \mathrm{ml}$.

RNA extraction and reverse transcription-quantitative polymerase chain reaction ( $R T-q P C R)$. Total RNA of treated and untreated UCMSCs was extracted using the RNeasy kit (Qiagen GmbH, Hilden, Germany) according to the manufacturer's protocol. ReverTra Ace kit (Toyobo Co., Ltd., Osaka, Japan) was used to perform the synthesis of cDNA with the following RT conditions: $65^{\circ} \mathrm{C}(5 \mathrm{~min}), 37^{\circ} \mathrm{C}(15 \mathrm{~min})$ and $98^{\circ} \mathrm{C}$ (5 min). qPCR was performed using RealMaster Mix SYBR Green (cat. no. FP202; Tiangen Biotech Co., Ltd., Beijing, China) in an iCycler iQ (Bio-Rad Laboratories, Inc., Hercules, CA, USA) under the following conditions: $95^{\circ} \mathrm{C}(30 \mathrm{sec}), 58^{\circ} \mathrm{C}$ (30 sec) and $72^{\circ} \mathrm{C}$ for $(30 \mathrm{sec})$, followed by a melt curve from $55-95^{\circ} \mathrm{C}$ in $0.5^{\circ} \mathrm{C}$ increments and $10 \mathrm{sec}$ intervals for 40 cycles. For quantification, GAPDH was used as the internal control while untreated UCMSC was negative control. The by $2^{-\Delta \Delta C q}$ method was used for relative quantification (20). The primers used in detection were listed in Table I. All detections of qPCR were performed three times.

Antibody array. Supernatants from treated and untreated groups were collected at $4 \mathrm{~h}$ post-stimulation. Supernatants were centrifuged $\left(800 \mathrm{x} \mathrm{g}, 10 \mathrm{~min}, 10^{\circ} \mathrm{C}\right)$ to remove the residual cells and then stored at $-80^{\circ} \mathrm{C}$. All samples, including TLR1 agonist treated and untreated, were screened for secreted protein using RayBio Human Antibody Array C Series 1000 (RayBiotech, Inc., Norcross, GA, USA) according to the manufacturer's protocol. Blots were analyzed using ImageJ software, version 1.50 (National Institutes of Health, Bethesda, MD, USA).

Leukocyte proliferation and leukocyte-mediated cytotoxicity detection. Peripheral blood mononuclear cells (PBMCs) were isolated from healthy volunteers and labeled by with carboxyfluorescein diacetate succinimidyl ester (CFSE) at $37^{\circ} \mathrm{C}$ for 10 min with a final concentration $10 \mu \mathrm{M}$. The current study was approved by the ethics committee of The Central Hospital of Dazhou (Dazhou, China), and written informed consent was obtained. The labeling reaction was stopped by adding $5 \mathrm{ml}$ pre-cooled complete medium. The remaining CFSE was removed by three washes with cold phosphate-buffered saline $\left(800 \times \mathrm{g}, 5 \mathrm{~min}, 4^{\circ} \mathrm{C}\right)$. PBMCs were then co-cultured with UCMSCs with or without TLR1/2 agonist Pam3Csk. The ratio of PBMCs and UCMSCs in co-culture system was 5:1. PBMCs were collected for proliferation detection by fluorescence-activated cell sorting (FACS) following 72-h stimulation.

Supernatants from the PBMC-UCSMSCs were harvested at 24,48 and $72 \mathrm{~h}$ post stimulation. Three centrifugation steps were performed to remove the remaining cells that may influence the detection. Release of lactate dehydrogenase (LDH) from injured cells was detected by a cytotoxicity kit according to the manufacturer's protocol. Cytotoxicity (\% lysis) was calculated using the following formula: $(\mathrm{E}-\mathrm{M}) /(\mathrm{T}-\mathrm{M}) \times 100$; $\mathrm{E}$ is the experimental release, $\mathrm{M}$ is the spontaneous release in the presence of media alone, and $\mathrm{T}$ is maximum release in the presence of $5 \%$ Triton X-100.

Detection of surface markers and co-stimulators detection by FACS. Pam3Csk treated and untreated UCMSCs were collected for FACS detection by following 72-h stimulation. The UCMSCs were fixed with $10 \%$ formaldehyde for $10 \mathrm{~min}$, then were stained 
with different antibodies to detect surface stem cells markers and co-stimulatory molecules. The assay was performed using CXP flow cytometry software, version 2.0 (Beckman Coulter, Inc. Brea, CA, USA). The positive and negative standard was gated according to the control groups. The antibodies used in detection are listed in Table II. The dilution of all antibodies in FACS assay was 1:100 and incubated $30 \mathrm{~min}$ at room temperature. All assays were conducted three times.

Differentiation detection of UCMSCs. Conditioned medium of chondrocytes (cat. no. A10071-01), osteocytes (cat. no. A10072-01) and adipocytes (cat. no. A10070-01) were obtained from Gibco (Thermo Fisher Scientific, Inc.) and added to UCMSCs $\left(1.5 \times 10^{5}\right.$ per well) in 6-well plates in the presence of $100 \mathrm{ng} / \mathrm{ml}$ Pam3Csk. Oil-red O for adipocytes, alizarin red for osteocytes and safranine staining for chondrocytes was conducted on day 5,14 and 20. Prior to staining [staining solutions diluted at 1:3 with double distilled water $\left.\left(\mathrm{ddH}_{2} \mathrm{O}\right)\right]$, cells were fixed with $10 \%$ formaldehyde soulution for $10 \mathrm{~min}$ at room temperature, then washed three times with $\mathrm{ddH}_{2} \mathrm{O}$. Subsequently, Mayer's hematoxylin staining was conducted for $5 \mathrm{~min}$, followed by three further washes with $\mathrm{ddH}_{2} \mathrm{O}$.

Statistical analysis. The analysis of RT-qPCR results was performed using Bio-Rad iQ5 software (Bio-Rad Laboratories, Inc.). Results are expressed as the mean \pm standard error and were analyzed with Student's t-test using SPSS software (version 16.0; SPSS, Inc., Chicago, IL, USA). P $<0.05$ was considered to indicate a statistically significant difference. All figures were created using GraphPad Prism 5 (GraphPad Software, Inc., La Jolla, CA, USA).

\section{Results}

TLR1/2 activation of UCMSCs increases the proliferation of PBMC and cytotoxicity effect. PBMCs from healthy volunteers were co-cultured with UCMSCs in the presence of $100 \mathrm{ng} / \mathrm{ml}$ Pam3Csk. The results indicated that the proliferation of PBMCs was higher following Pam3Csk stimulation (20.7\%) in PBMC-UCMSCs co-culture system compared with the untreated group (10.2\%; Fig. 1). The results also demonstrated that Pam3Csk only led to $10.2 \%$ proliferation rate in PBMC. The results suggested that activation of TLR $1 / 2$ pathway in UCMSCs increases the immune response.

The immune attack was measured by detecting the LDH levels in culture supernatants from injured cells, which is a classical method for measuring leukocyte-mediated cytotoxicity. In the current study, PBMCs were co-cultured with UCMSCs and Pam3Csk was added to activate TLR1/2 signaling. The negative was PBMCs-UCMSCs without Pam3Csk. The results indicated no difference significant difference between the two groups at $24 \mathrm{~h}$ post-co-culture $(10.3 \%$ vs. $11.7 \%$ : $\mathrm{P}=0.265$ ), whereas LDH levels were significantly increased in the Pam3Csk treatment group compared with the untreated group at $48 \mathrm{~h}(12.9 \%$ vs. $23.9 \% ; \mathrm{P}=0.01)$ and $72 \mathrm{~h}(22.3 \%$ vs. $32.7 \%$; $\mathrm{P}=0.037$ ) post-co-culture (Table III).

Activation of TLR1/2 signaling increases the surface expression of co-stimulators of UCMSCs. The data of the current
Table II. Monoclonal antibodies used for fluorescence-activated cell sorting analysis.

\begin{tabular}{ccc}
\hline Name & Company & Cat no. \\
\hline CD40 & $\begin{array}{c}\text { eBioscience, Inc. } \\
\text { (San Diego, CA, USA) }\end{array}$ & $17-9953$ \\
CD80 & eBioscience, Inc. & $11-0809$ \\
CD86 & eBioscience, Inc. & $12-0869$ \\
CD59 & eBioscience, Inc. & $11-0596$ \\
CD74 & eBioscience, Inc. & $11-0748$ \\
CD90 & eBioscience, Inc. & $45-0909$ \\
\hline
\end{tabular}

$\mathrm{CD}$, cluster of differentiation.

Table III. Lactate dehydrogenase levels in the supernatant of umbilical cord MSC-PBMC co-culture system.

\begin{tabular}{cccc}
\hline Time & MSC + PBMC & MSC + PBMC + Pam3Csk & P-value \\
\hline $24 \mathrm{~h}$ & $10.3 \pm 2.2 \%$ & $11.7 \pm 2.8 \%$ & 0.265 \\
$48 \mathrm{~h}$ & $13.0 \pm 1.6 \%$ & $23.9 \pm 5.1 \%$ & $<0.05^{\mathrm{a}}$ \\
$72 \mathrm{~h}$ & $22.3 \pm 3.3 \%$ & $32.7 \pm 4.5 \%$ & $<0.05^{\mathrm{a}}$ \\
\hline
\end{tabular}

${ }^{a} \mathrm{P}<0.05$ vs. control group. Results are expressed as mean values \pm standard error of the mean. MSC, mesenchymal stem cell; PBMC, pheripheral blood mononuclear cell.

study indicated that TLR1/2 agonist induces immune attack and causes injury to UCMSCs. Thus, the study subsequently examined the effect of activation of TLR $1 / 2$ signaling on the UCMSCs surface expression of co-stimulators, which are important for mediating immune responses. The results indicated that CD80 (11.5 vs. $1.1 \%$ ) and CD86 (12.4 vs. $2.0 \%)$ were significantly upregulated $(\mathrm{P}=0.036$ and $\mathrm{P}=0.043$, respectively) in UCMSCs treated with Pam3Csk compared with the control group (Fig. 2A). The expression variation of specific markers of UCMSCs were also detected and the results indicated that CD59 (98.2 vs. 96.6\%) and CD74 (98.6 vs. 98.8\%) and CD90 (89.8 vs. $98.6 \%$ ) levels were marginally inhibited following Pam3Csk stimulation compared with untreated cells (Fig. 2B). The FACS results indicated that activation of TLR1/2 altered the surface expression of co-stimulators of UCMSCs, however the effect was not marked.

Immune-modulation molecules were upregulated in the presence of Pam3Csk. The UCMSCs were stimulated with TLR1/2 agonist, Pam3Csk, and the expression of pro-inflammatory cytokines (IFN- $\beta$, IL- 6 , IL- 8 and TNF- $\alpha$ ), chemokines (CCL-5, MCP-1, IP-10 and MIP-1 $\alpha$ ) and stem cell markers (Nanog, Sox 2, Lin28 and Otx2) were examined at 4, 12, 24, 72 and $120 \mathrm{~h}$ following agonist treatment. It was demonstrated that IL-6, CCL-5, IP-10 and MIP-1 $\alpha$ were significantly induced to high expression levels upon Pam3Csk stimulation compared with the control (Fig. 3A and B). Additionally, it was observed that although IFN- $\beta$, IL- 8 , TNF- $\alpha$ and MCP-1 expression levels were significantly induced in the presence 


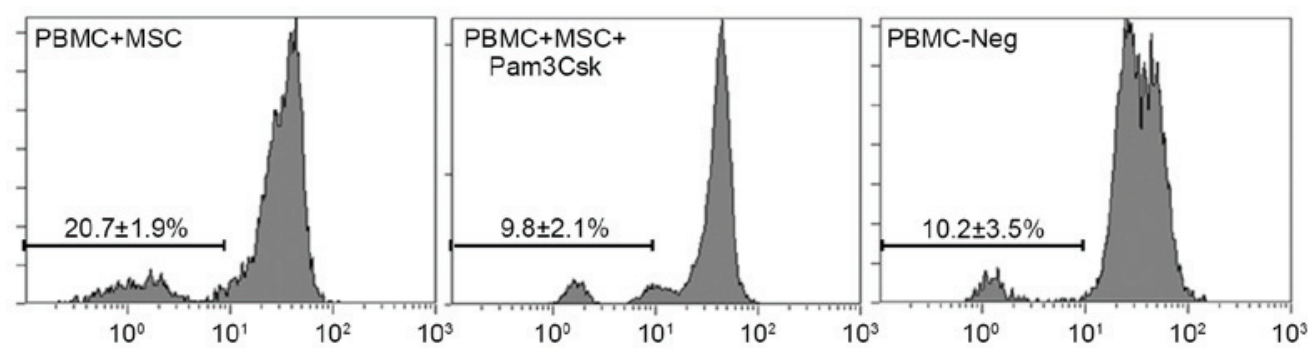

Figure 1. Toll-like receptor 1/2 agonist increase the proliferation of allogeneic PBMCs in a PBMC-umbilical cord MSC co-culture system. PBMC, peripheral blood mononuclear cell; MSC, mesenchymal stem cell.

A
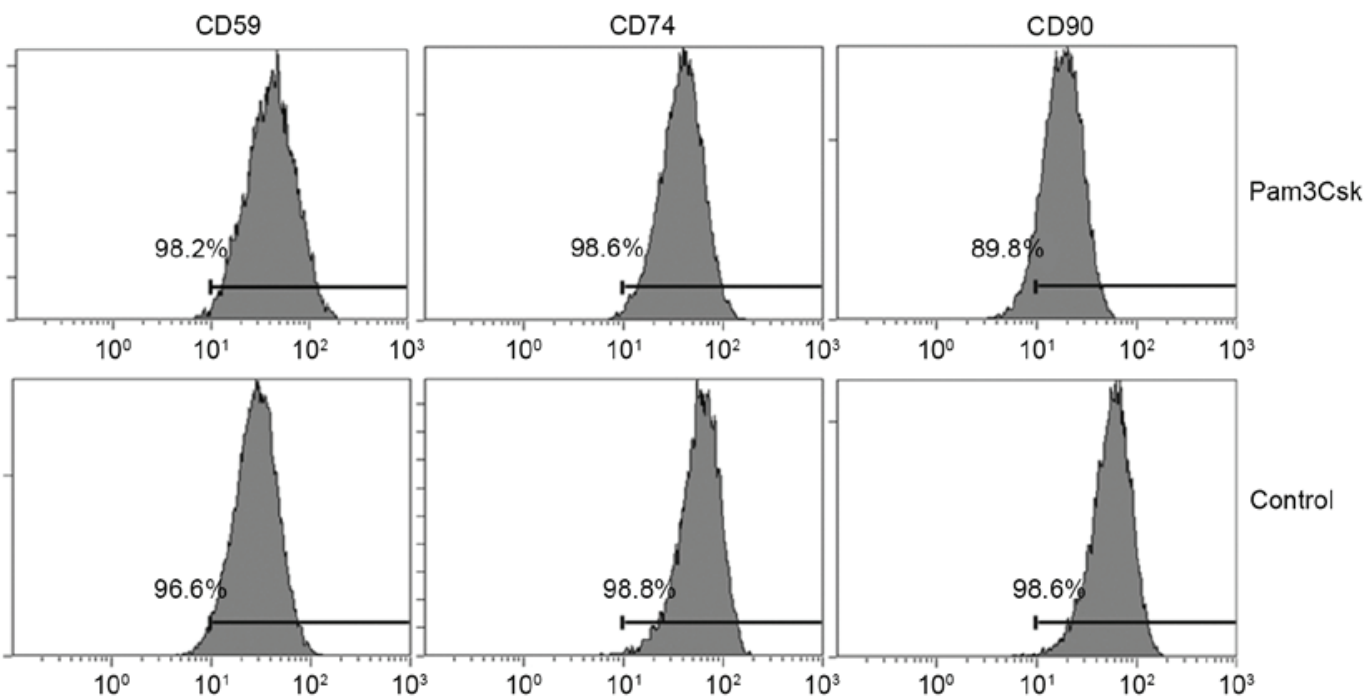

B
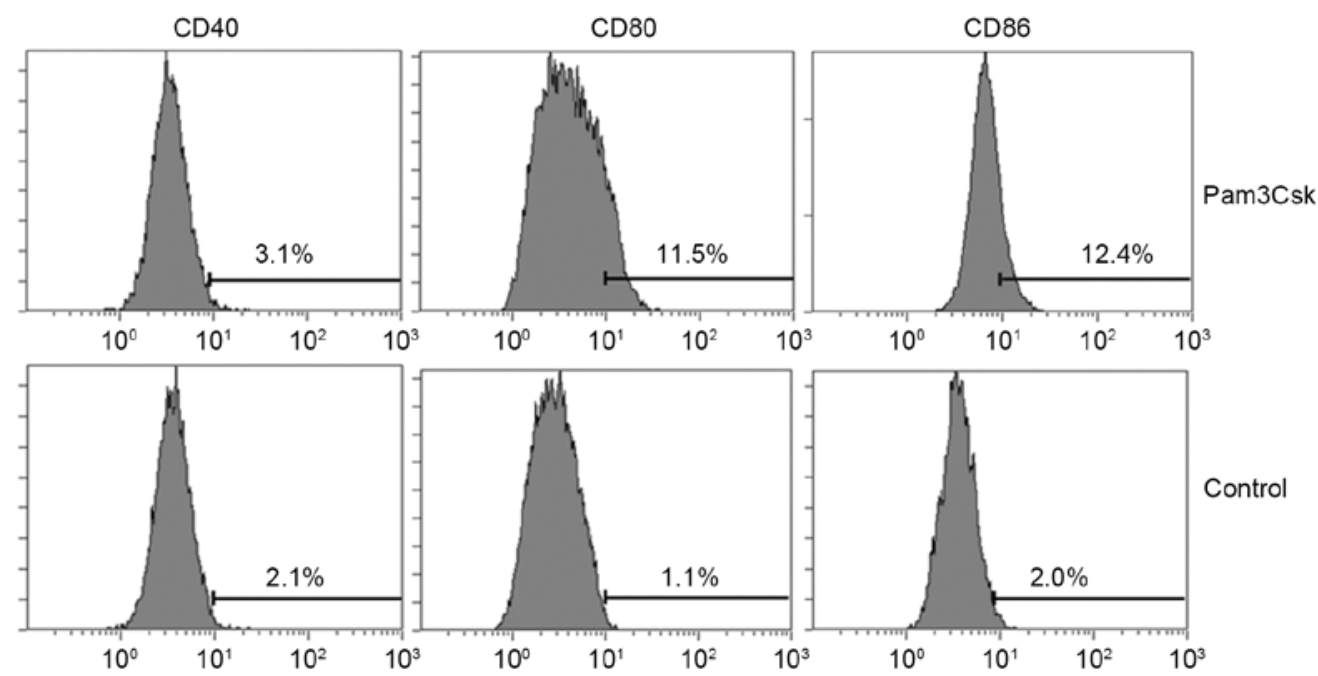

Figure 2. Pam3Csk induced the expression of co-stimulatory factors in umbilical cord mesenchymal stem cells, however had no influence on stem cell markers (A) Stem cell markers, (B) co-stimulatory factors.

of Pam3Csk compared with the control, the expression levels decreased markedly at the later time points (Fig. 3A and B).

Additionally, the expression levels of stem cells markers were examined to determine whether activation of Pam3Csk affects the stemness of UCMSCs. The present study demonstrated that the expression level of Nanog was significantly inhibited following Pam3Csk stimulation at $12 \mathrm{~h}$ compared with control levels $(\mathrm{P}=0.021)$, whereas Sox 2 levels were only inhibited compared with the control at $120 \mathrm{~h}$ treatment
$(\mathrm{P}=0.028$; Fig. 3C). It was also observed that the expression levels of Lin 28 and Otx2 were not altered in the presence of Pam3Csk (Fig. 3C). Thus, the activation of TLR1/2 signaling upregulated the expression of pro-inflammatory molecules and may inhibit the stemness maintenance of UCMSCs.

Pam3Csk increases the secretion of pro-inflammatory cytokines in UCMSCs. The secretion of pro-inflammatory cytokines in the supernatants of the Pam3Csk-treated and 
A

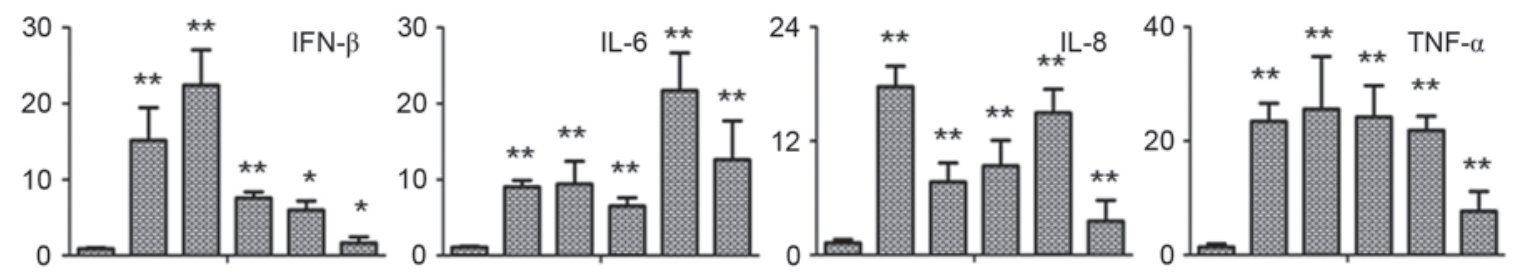

B
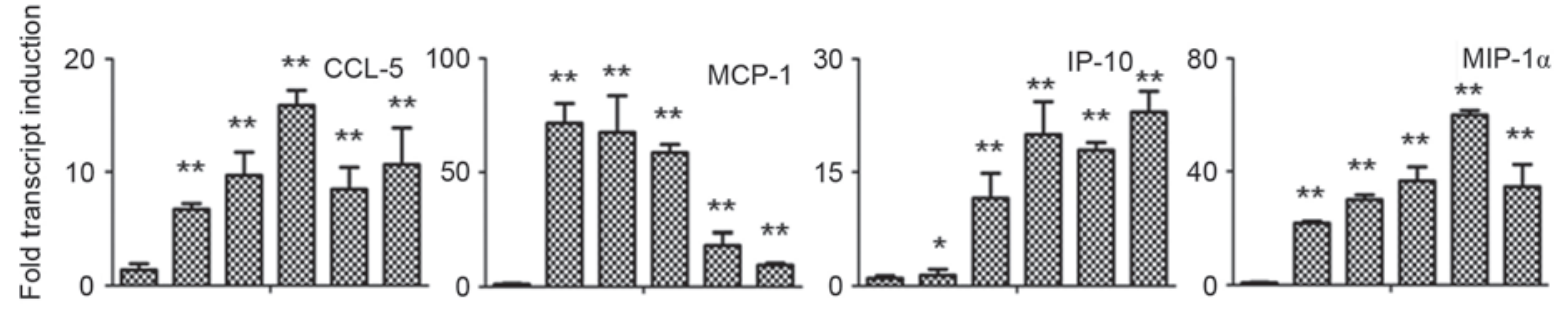

C

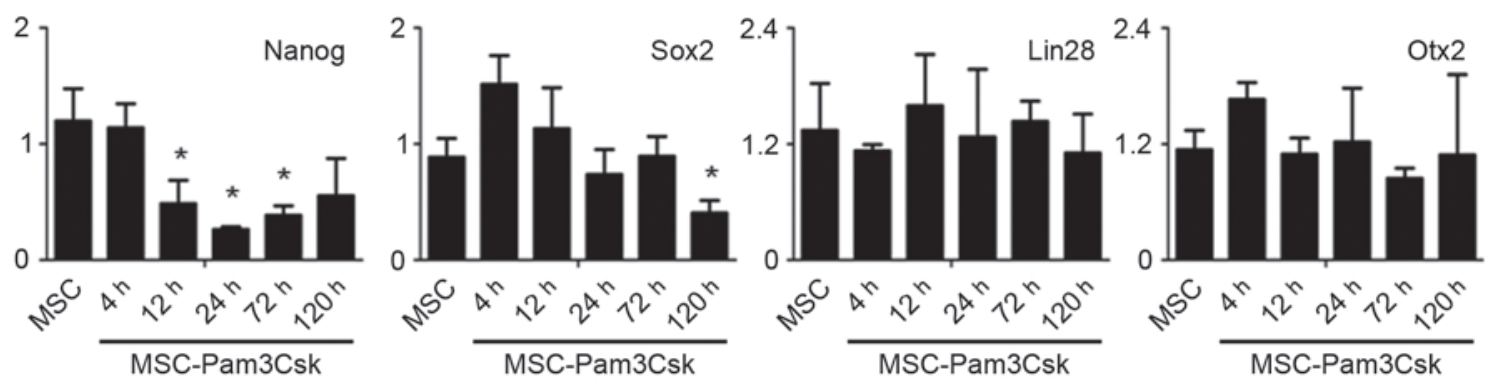

Figure 3. Secretion of pro-inflammatory molecules was significantly increased in UCMSCs treated with Pam3Csk. The mRNA levels of (A) cytokines, (B) chemokines and (C) stem cell markers were measured. ${ }^{*} \mathrm{P}<0.05,{ }^{* *} \mathrm{P}<0.001$ vs. control group. Results are expressed as mean values \pm standard error of the mean. IFN- $\beta$, interferon- $\beta$; IL, interleukin; TNF- $\alpha$, tumor necrosis factor- $\alpha$; CCL5, C-C motif chemokine ligand 5; MCP, monocyte chemoattractant protein; IP10, interferon $\gamma$-induced protein 10; MIP-1 $\alpha$, macrophage inflammatory protein-1 $\alpha$; Nanog, Nanog homeobox; SOX2, sex determining region Y-box 2; Lin28, Lin-28 homolog A; Otx2, orthodenticle homeobox 2.

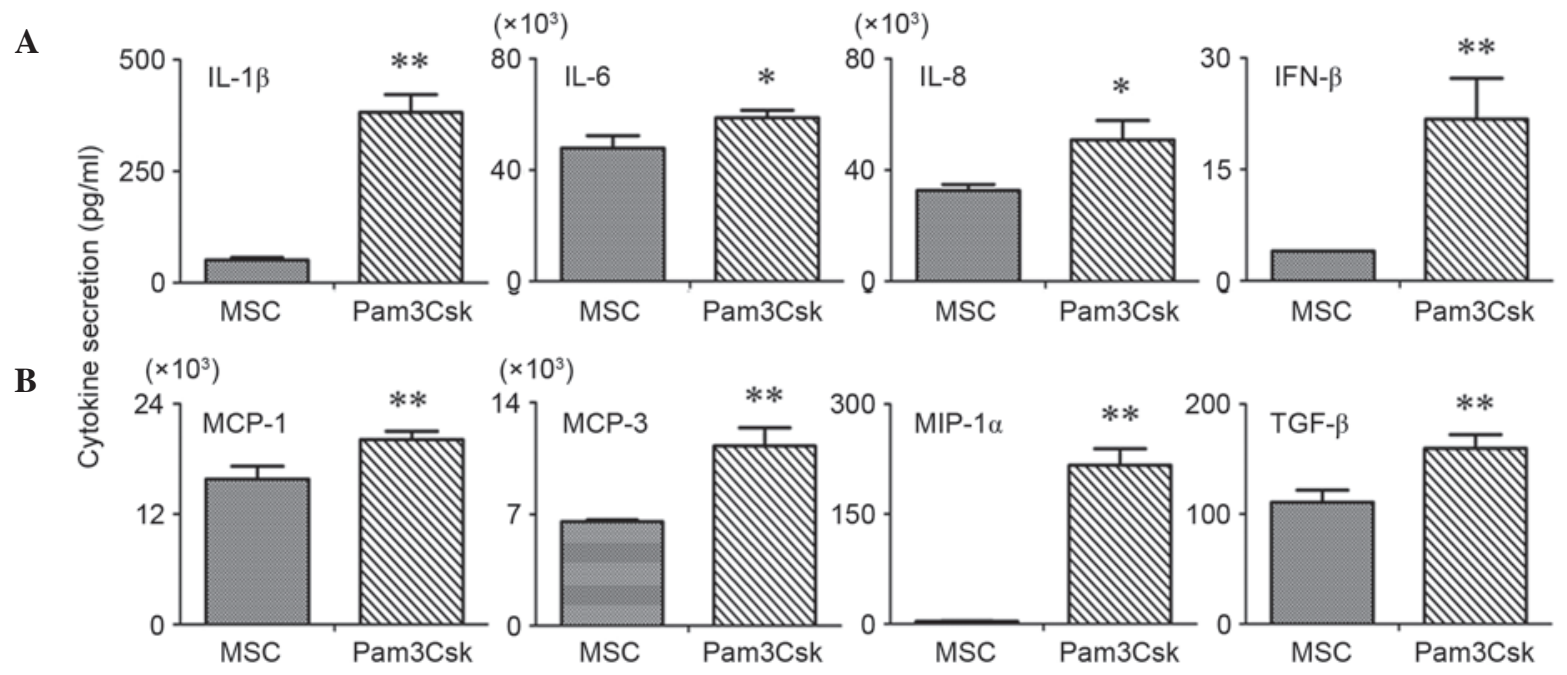

Figure 4. Toll-like receptor 1/2 agonist upregulates the expression levels of co-stimulatory molecules in umbilical cord MSCs. The levels of (A) co-stimulatory molecules and (B) surface markers were measured. ${ }^{*} \mathrm{P}<0.05,{ }^{* *} \mathrm{P}<0.001$ vs. MSC. Results are expressed as mean values \pm standard error of the mean. IL, interleukin, IFN- $\beta$, interferon- $\beta$; MSC, mesenchymal stem cell; MCP, monocyte chemoattractant protein; MIP-1 $\alpha$, macrophage inflammatory protein-1 $\alpha$; TGF- $\beta$, tumor growth factor- $\beta$.

untreated UCMSCs was measured using a RayBio antibody chip. The results indicated that IL-1 $\beta$, IFN- $\beta$, MCP-1, MCP-3, MIP- $1 \alpha$ and TGF- $\beta$ were significantly upregulated in the supernatants of Pam3Csk treated UCMSCs compared with untreated UCMSCs $(\mathrm{P}<0.001$; Fig. 4). Additionally, IL-6 and IL-8 levels were significantly induced upon TLR1/2 agonist stimulation compared with controls $(\mathrm{P}=0.032$ and $\mathrm{P}=0.029$, respectively), but not as markedly as MCP-1, MCP-3, etc (Fig. 4).

Pam3Csk stimulation had no effect on the differentiation ability of UCMSCS. A previous study indicated that differentiation of UCMSCs alters the immune status and increases immune responses (21). Thus, aimed to 
A
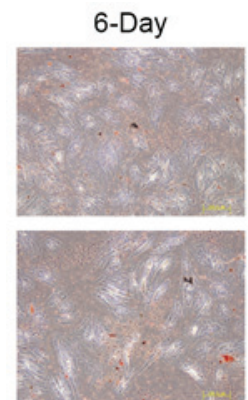

B
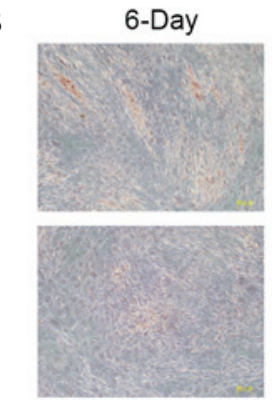

6-Day

C
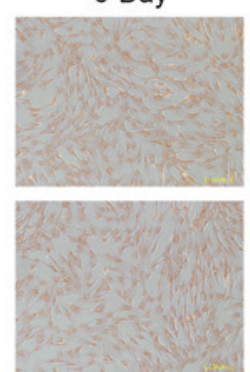

14-Day

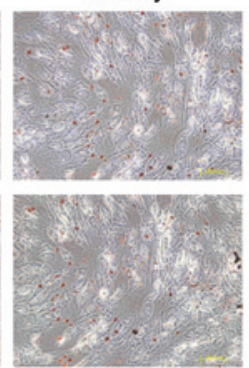

14-Day
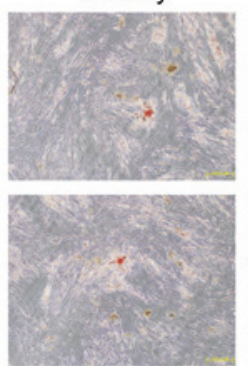

14-Day
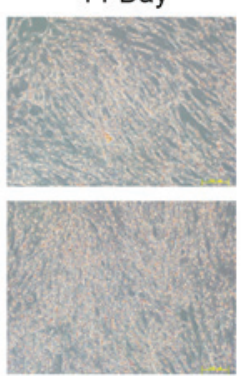

20-Day

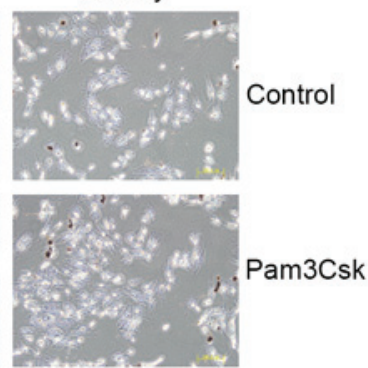

20-Day

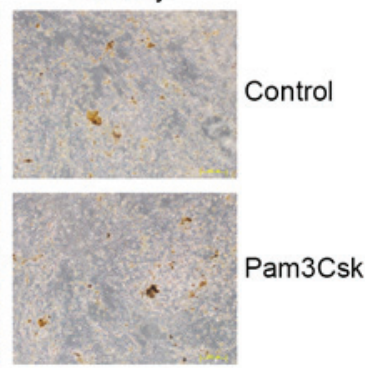

20-Day

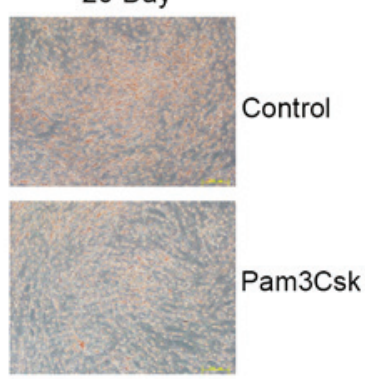

Figure 5. Pam3Csk treatment had no influence on the differentiation ability of UCMSCs. UCMSCs were stimulated to differenctate into (A) adipocytes (oil-red O staining, x400 magnification), (B) osteoblasts (alizarin red staining, x200 magnification) and (C) chondrocytes (safranine staining, x200 magnification). UCMSCs, umbilical cord mesenchymal stem cells.

determine whether TLR1/2 activation alters the differentiation of UCMSCs. The conditioned media for adipocyte, osteoblast and chondrocyte differentiation were added to UCMSCs following stimulation with Pam3Csk to detect the importance of TLR1/2 on UCMSCs differentiation ability. At day 6, 14 and 20 post-stimulation, alizarin red for osteoblasts, safranine for chondrocytes and oil-red $\mathrm{O}$ staining for adipocytes was conducted to assess the differentiation of UCMSCs. The results indicated that activation of TLR $1 / 2$ by Pam3Csk stimulation exerted no observable effect on the differentiation UCMSCs to adipocytes, osteoblasts and chondrocytes (Fig. 5).

\section{Discussion}

Multiple differentiation and self-renewal properties of MSCs enable its usage in clinical cell-based therapies (21). MSC differentiate into cell types for various tissues, including bone, cartilage, adipocytes, connective stomal cells, hepatocytes and muscle (22). In addition to differentiating into specific cells types, MSCs are also involved in tissue regeneration due to their trophic effects (23). Thus, knowledge of molecules, and mechanisms, that regulate the properties and potential immunogenicity of MSCs is important for the therapeutic use of MSCs. Immunomodulatory properties enable MSCs to suppress the activation and proliferation of $\mathrm{T}$ and $\mathrm{B}$ cell responses, and to interfere with the maturation of dendritic and NK cells (7-9). However, previous studies indicated that the in vivo microenvironment alters the immune status, enhances immune responses and causes to failure of MSC-based therapy (10-12).

TLR is the most important (PAMP) family, with an important role in defending against invading pathogens (15). Among the 11 members of the human TLR family, TLR1/2, which is located on the cell surface and recognized by gram-positive bacteria, is involved in the recognition of a variety of microbial components, including lipoproteins. Previous research demonstrated that activation of TLR $1 / 2$ exhibited no effect on the immune status of MSC from bone marrow (17), while no studies focussing upon the role of TLR $1 / 2$ in regulating the immune status of MSCs from the umbilical cord have been conducted. As UCMSCs attract attention in cell-based therapy, it is important to analyze whether activation of TLR $1 / 2$ pathway may alter the immunogenicity of UCMSCs. (24). The current study demonstrated that activation of TLR $1 / 2$ signaling in UCMSCs promoted immunogenicity by increasing the proliferation of PBMC in co-culture with UCMSCs and enhancing the release of LDH into the supernatant of the PBMC-UCMSCs 
co-culture system. In support of this observation, the treatment of Pam3Csk also upregulated the expression of surface co-stimulators, CD80 and CD86, to a certain extent, however Pam3Csk exhibited no obvious influence on the levels of stem cell markers, including CD59, CD74 and CD90. Antibody array chip and RT-qPCR analysis was also performed. The antibody chip array detecting secretion of pro-inflammatory molecules indicated the levels of IL-1 $\beta$, INF- $\beta$, MCP-1, MCP-3, MIP- $1 \alpha$ and TGF- $\beta$ in the supernatants of Pam3Csk-treated UCMSCs were significantly increased. RT-qPCR for gene expression levels also indicated that the expression of various pro-inflammatory cytokines (INF- $\beta$, IL- 6 , IL- 8 and TNF- $\alpha$ ) and chemokines (CCL-5, MCP-1, IP-10 and MIP-1 $\alpha$ ) was increased by Pam3Csk. Huang et al (21) suggested that MSCs lost the immune privilege properties when differentiated into cardiac cells and finally resulted in the rejection of engrafted MSCs. Thus, the present study aimed to establish whether the enhanced immune status was associated with alteration of the differentiation abilities in UCMSCs. Conditioned media were introduced for adipocyte, osteoblast and chondrocyte differentiation of UCMSCs upon stimulation with Pam3Csk, however, no observable change in differentiation ability was detected following activation of TLR $1 / 2$ signaling with Pam3Csk.

In clinical trials, numerous endogenous ligands, including heparin sulfate, oxidized low-density lipoprotein, uric acid and heat shock proteins have been previously demonstrated to activate TLRs. These endogenous TLR agonists may regulate functions of UCMSCs by endogenous stimuli during tissue repair. Future studies are required to study the regulatory mechanisms of the biological functions of UCMSCs. The current study firstly confirmed that activation of the TLR1/2 pathway increased the immunogenicity of UCMSCs. In clinical cell-based therapy, the engrafted MSCs encountered numerous endogenous ligands which may activate TLR pathways. Thus, the present study identified the potential risks of the use of MSCs in clinical therapy

\section{References}

1. Le Blanc K, Frassoni F, Ball L, Locatelli F, Roelofs H, Lewis I, Lanino E, Sundberg B, Bernardo ME, Remberger M, et al: Mesenchymal stem cells for treatment of steroid-resistant, severe, acute graft-versus-host disease: A phase II study. Lancet 371: 1579-1586, 2008.

2. Kharaziha P, Hellström PM, Noorinayer B, Farzaneh F, Aghajani K, Jafari F, Telkabadi M, Atashi A, Honardoost M, Zali MR and Soleimani M: Improvement of liver function in liver cirrhosis patients after autologous mesenchymal stem cell injection: A phase-II clinical trials. Eur J Gastroenterol Hepatol 21: 1199-1205, 2009.

3. Vojtassák J, Danisovic L, Kubes M, Bakos D, Jarábek L, Ulicná M and Blasko M: Autologous biograft and mesenchymal stem cells in treatment of the diabetic foot. Neuro Endocrinol Lett 27 (Suppl 2): S134-S137, 2006.

4. Guiducci S, Porta F, Saccardi R, Guidi S, Ibba-Manneschi L, Manetti M, Mazzanti B, Dal Pozzo S, Milia AF, Bellando-Randone S, et al: Autologus mesenchymal stem cells foster revascularization of ischemic limbs in systemic sclerosis: A case report. Ann Intern Med 153: 650-654, 2010.
5. Phinney DG and Prockop DJ: Concise review: Mesenchymal stem/multipotent stromal cells: The state of transdifferentiation and modes of tissue repair-current views. Stem Cells 25: 2896-2902, 2007.

6. Bordignon C, Carlo-Stella C, Colombo MP, De Vincentiis A, Lanata L, Lemoli RM, Locatelli F, Olivieri A, Rondelli D, Zanon P and Tura S: Cell therapy: Achievements and perspectives. Haematologica 84: 1110-1149, 2011.

7. Bassi EJ, Aita CA and Câmara NO: Immune regulatory properties of multipotent mesenchymal stromal cells: Where do we stand? World J Stem Cells 3: 1-8, 2011.

8. Shi M, Liu ZW and Wang FS: Immunomodulatory properties and therapeutic application of mesenchymal stem cells. Clin Exp Immunol 164: 1-8, 2011

9. Han KH, Ro H, Hong JH, Lee EM, Cho B, Yeom HJ, Kim MG, Oh KH, Ahn C and Yang J: Immunosuppressive mechanisms of embryonic stem cells and mesenchymal stem cells in alloimmune response. Transpl Immunol 25: 7-15, 2011.

10. Li Y and Lin F: Mesenchymal stem cells are injured by complement after their contact with serum. Blood 120: 3436-3443, 2012.

11. Allison M: Genzyme backs Osiris, despite prochymal flop. Nat Biotechnol 27: 966-967, 2009.

12. Spaggiari GM, Capobianco A, Becchetti S, Mingari MC and Moretta L: Mesenchymal stem cell-natural killer cell interactions: Evidence that activated NK cells are capable of killing MSCs, whereas MSCs can inhibit IL-2-induced NK-cell proliferation. Blood 107: 1484-1490, 2006.

13. Akira S, Uematsu S and Takeuchi O: Pathogen recognition and innate immunity. Cell 124: 783-801, 2006.

14. Blasius AL and Beutler B: Intracellular toll-like receptors. Immunity 32: 305-315, 2010.

15. Opitz CA, Litzenburger UM, Lutz C, Lanz TV, Tritschler I, Köppel A, Tolosa E, Hoberg M, Anderl J, Aicher WK, et al: Toll-like receptor engagement enhances the immunosuppressive properties of human bone marrow-derived mesenchymal stem cells by inducing indoleamine-2,3-dioxygenase-1 via interferon-beta and protein kinase R. Stem Cell 27: 909-919, 2009.

16. Pevsner-Fischer M, Morad V, Cohen-Sfady M, Rousso-Noori L, Zanin-Zhorov A, Cohen S, Cohen IR and Zipori D: Toll-like receptors and their ligands control mesenchymal stem cell function. Blood 109: 1422-1432, 2007.

17. DelaRosa O and Lombardo E: Modulation of adult mesenchymal stem cells activity by toll-like receptors: Implications on therapeutic potential. Mediators Inflamm 2010: 865601, 2010.

18. Zhang L, Liu D, Pu D, Wang Y, Li L, He Y, Li Y, Li L and Li W: The TLR7 agonist imiquimod promote the immunogenicity of msenchymal stem cells. Biol Res 48: 6, 2015.

19. Zhang L, Liu D, Pu D, Wang Y, Li L, He Y, Li Y, Li L, Qiu Z, Zhao $\mathrm{S}$ and $\mathrm{Li} \mathrm{W}$ : The role of toll-like receptor 3 and 4 in regulating the function of mesenchymal stem cells isolated from umbilical cord. Int J Mol Med 35: 1003-1010, 2015.

20. Arocho A, Chen B, Ladanyi M and Pan Q: Validation of the 2-DeltaDeltaCt calculation as an alternate method of data analysis for quantitative PCR of BCR-ABL P210 transcripts. Diag Mol Path 15: 56-61, 2006.

21. Huang XP, Sun Z, Miyagi Y, McDonald Kinkaid H, Zhang L, Weisel RD and Li RK: Differentiation of allogeneic mesenchymal stem cells induces immunogenicity and limits their long-term benefits for myocardial repair. Circulation 122: 2419-2429, 2010.

22. Pittenger MF, Mackay AM, Beck SC, Jaiswal RK, Douglas R, Mosca JD, Moorman MA, Simonetti DW, Craig S and Marshak DR: Multilineage potential of adult human mesenchymal stem cells. Science 284: 143-147, 1999.

23. Augello A, Kurth TB and De Bari C: Mesenchymal stem cells: A perspective from in vitro cultures to in vivo migration and niches. Eur Cell Mater 20: 121-133, 2010.

24. Hsieh JY, Wang HW, Chang SJ, Liao KH, Lee IH, Lin WS, Wu CH, Lin WY and Cheng SM: Mesenchymal stem cells from human umbilical cord express preferentially secreted factors related to neuroprotection, neurogenesis, and angiogenesis. PLoS One 8: e72604, 2013. 